\title{
Carbon ions beam therapy monitoring with the INSIDE in-beam PET
}

Francesco Pennazio', Giuseppe Battistoni ${ }^{2}$, Maria Giuseppina Bisogni ${ }^{3,4}$, Niccolò Camarlinghi $^{3,4}$, Alfredo Ferrari ${ }^{5}$, Veronica Ferrero ${ }^{1,6}$, Elisa Fiorina ${ }^{1}$, Matteo Morrocchi ${ }^{3,4}$, Paola Sala ${ }^{2}$, Giancarlo Sportelli, ${ }^{3,4}$, Richard Wheadon ${ }^{1}$, Piergiorgio Cerello ${ }^{1}$.

1. INFN - Torino

2. INFN - Milano

3. Università di Pisa

4. INFN - Pisa

5. CERN

6. Università degli Studi di Torino

In-vivo range monitoring techniques are necessary in order to fully take advantage of the high dose gradients deliverable in hadrontherapy treatments. Positron Emission Tomography (PET) scanners can be used to monitor beam-induced activation in tissues and hence measure the range. The INSIDE (Innovative Solutions for In-beam DosimEtry in Hadrontherapy) in-beam PET scanner, installed at the Italian National Center of Oncological Hadrontherapy (CNAO, Pavia, Italy) synchrotron facility, has already been successfully tested in-vivo during a proton therapy treatment.

We discuss here the system performance evaluation with carbon ion beams, in view of future in-vivo tests. The work is focused on the analysis of activity images obtained with therapeutic treatments delivered to polymethyl methacrylate (PMMA) phantoms, as well as on the test of an innovative and robust Monte Carlo simulation technique for the production of reliable prior activity maps.

Images are reconstructed using different integration intervals, so as to monitor the activity evolution during and after the treatment. Three procedures to compare activity images are presented, namely Pearson Correlation Coefficient, Beam's Eye View and Overall View. Images of repeated irradiations of the same treatments are compared to assess the integration time necessary to provide reproducible images. The range agreement between simulated and experimental images is also evaluated, so as to validate the simulation capability to provide sound prior information.

The results indicate that at treatment end, or at most $20 \mathrm{~s}$ afterwards, the range measurement is reliable within 1-2 $\mathrm{mm}$, when comparing both different experimental sessions and data with simulations.

In conclusion, this work shows that the INSIDE in-beam PET scanner performance is promising towards its in-vivo test with carbon ions.

\section{Introduction}

Treatment monitoring in hadron-therapy is one of the key issues for improving the treatment quality assurance procedures, especially for hypo-fractionated planning [1-3]. 
The INSIDE collaboration has built a treatment monitoring system based on a dual-head PET scanner and a charged particles tracker used as dose profiler. The PET scanner is installed at the Italian National Center of Oncological Hadrontherapy (CNAO, Pavia, Italy), the only dual-ion (proton and carbon ions) facility in Italy [4], and it is now going through a test program. The PET scanner performance has already been tested in-vivo [5], during proton treatment sessions. In this work, we will focus on the performance of the PET scanner as monitoring system during carbon ion treatments.

The treatment accuracy assessment by means of PET is not straightforward. The measured activity does not match the shape of the delivered dose for any of the primary ion species used in hadrontherapy [6,7]. Hence, the possibility of reconstructing the delivered dose starting from the measured activity is still an open issue. However, the expected 3D shape of the activity can be derived from the delivered dose according to the treatment plan, either analytically [8] or by means of Monte Carlo simulations (MC), and then compared to that induced by the actual treatment, so that their agreement can be quantified. In this work we investigate how to generate a prior image by means of FLUKA-based $[9,10]$ MC simulation and how to perform the comparison with the measured image. Although the proposed methods are straightforwardly applicable to protons and other ions, in this work we will focus on the case of carbon ions beam therapy.

The PET experimental data, required to assess the treatment accuracy, can be acquired by making use of several PET-based monitoring techniques [11, 12]: during the treatment (in-beam PET) [13-15], immediately after the treatment with an in-room dedicated scanner (in-room PET) [16], or with a delay of several minutes with a conventional scanner (off-line PET) [17]. The main advantage of in-beam PET with respect to the after-treatment techniques is the capability to perform on-line monitoring during the treatment and hence provide an early treatment quality feedback: in such conditions, the comparison between measured and expected images can be more precise, since it includes the activity evolution during the treatment and not just its final state.

We will discuss, for a carbon ion beam therapy fraction delivered on a homogeneous PMMA phantom, the comparison of prior (i.e. simulated) versus measured activity during the treatment delivery, with two main goals:

- evaluating the capability of the INSIDE in-beam PET scanner to provide an assessment of the treatment quality as early as possible with respect to the treatment start;

- evaluating the reliability of Monte Carlo simulations in providing a prior image to compare with the measurements.

This paper is organised as follows: in the first part a description of the INSIDE in-beam PET scanner and of the experimental measurements is presented. The procedure implemented in Monte Carlo simulation is then described, with a special focus on the most common pitfalls that may jeopardize the application of MC to the generation of in-beam PET images. The methods implemented to post-process and compare images are also presented in detail. In the second part the results obtained from measurements and simulations are discussed. The images obtained from the repetition of the same irradiation are compared so as to assess the imaging repeatability and to characterise the measurement feasibility and stability during and immediately after the treatment. The images obtained from experimental measurements are also compared with the $\mathrm{MC}$ ones, in order to evaluate the reliability of MC simulation 
technique proposed in providing a prior image. In the last part the implications of the results on in-vivo carbon ion treatment monitoring are discussed.

\section{Materials and methods}

\subsection{The INSIDE in-beam PET}

The INSIDE in-beam PET scanner (fig. 1a,b) [18] is based on small Lutetium Fine Silicate (LFS) scintillator elements individually coupled to Silicon PhotoMultipliers (SiPM). The scintillator element pitch and thickness are $3.2 \mathrm{~mm}$ and $20 \mathrm{~mm}$, respectively; the PET detector blocks feature $16 \times 16$ elements. The scanner is composed by two planar heads, including 10 blocks each, so that their active area is about $100 \times 250 \mathrm{~mm}^{2}$. Heads distance in this work was $50 \mathrm{~cm}$, hence the geometrical acceptance was $250 \mathrm{~mm}$ along the axial $(z)$ direction and 23 degrees in the radial $(x-y)$ plane.

The front-end electronics is based on the TOFPET ASIC [19], which features Time To Digit Coverter (TDC) for the Time Of Flight (TOF) measurement and Time Over Threshold (TOT) for the energy measurement. Each 64-channel ASIC is controlled and read out by means of a Xlilinx Virtex FPGA, which encodes the data in a convenient format and sends them to a HP Proliant server through Gigabit Ethernet links. The server implements the time sorting, the coincidence finding and performs the on-the-fly image reconstruction, to obtain the 3D spatial distribution of the measured activity. The reconstructed FOV is $224 \times 112 \times 264 \mathrm{~mm}^{3}$, segmented into voxels of $1.6 \times 1.6 \times 1.6 \mathrm{~mm}^{3}$.

The implemented image reconstruction algorithm is the iterative Maximum Likelihood Expectation Maximization (MLEM) [20]. One advantage of the MLEM approach over the analytical ones consists in the possibility of modeling detectors with incomplete angular sampling, as the geometry of the system and other physical effects can be encoded in the so-called system response matrix. In this work, the system response matrix is computed using a Siddon multi-ray algorithm, as described in [21]. Moreover, symmetries in the system matrix are exploited so as to reduce its memory footprint.

The algorithm has been described and tested with a similar geometry [22]. Consistently with other works, the iterative MLEM algorithm was early-stopped after the fifth iteration, in order to avoid unwanted noise build-up caused by low statistics and to reduce computation time, which is a key to obtain an on-the-fly response $[5,18,22]$. The TOF information is presently not exploited in image reconstruction. 


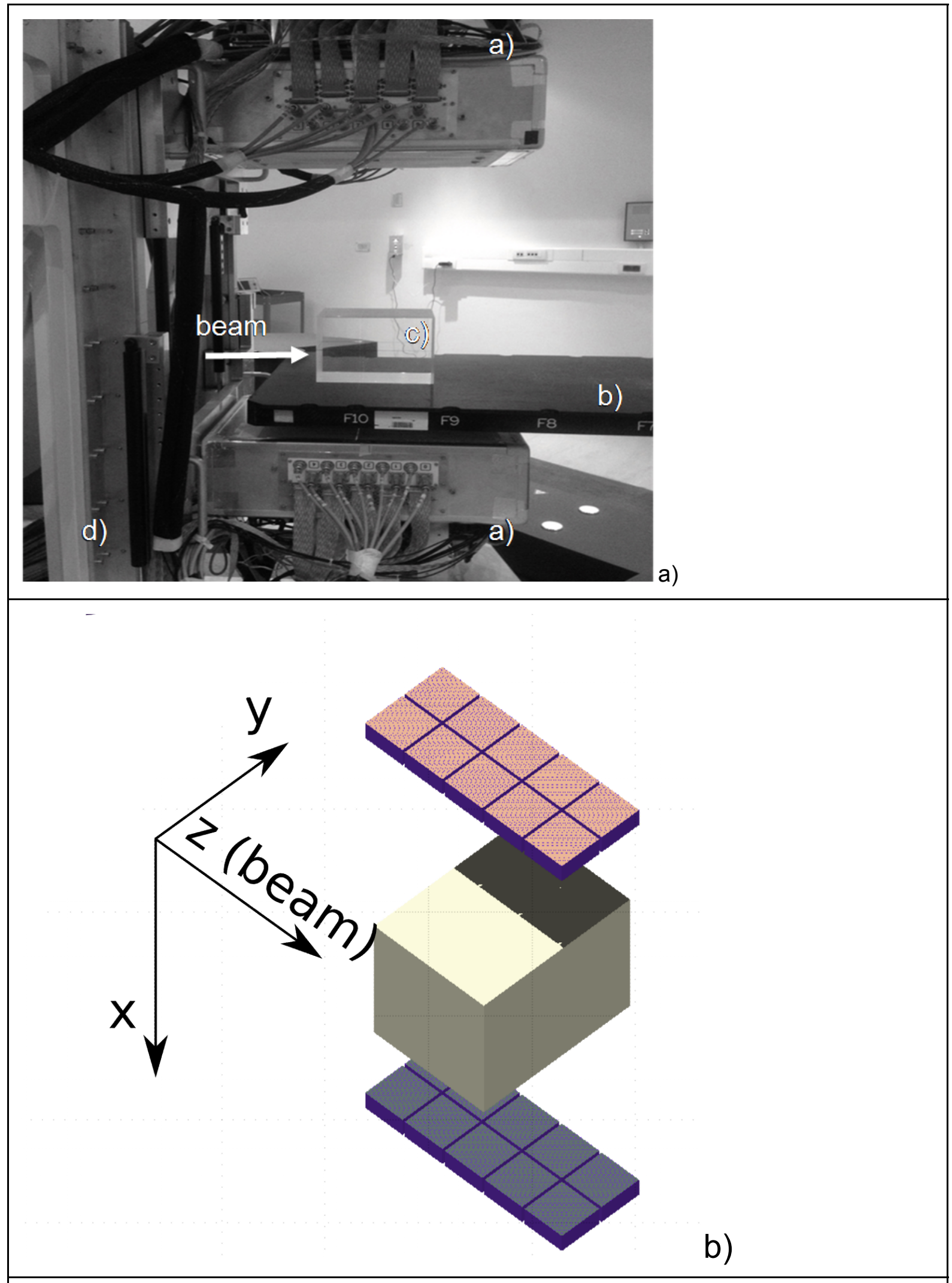

Fig. 1. a) The INSIDE scanner in a CNAO treatment room. The labelled components are a) the PET heads; b) the patient bed; c) the PMMA target; d) the scanner mobile support. b) Scheme of detectors, PMMA phantom and axis. The beam is coming from the negative $z$ direction. 


\subsection{Experimental measurements}

The experimental measurements were performed during a test session of the INSIDE scanner at CNAO (fig. 1a), with a carbon ion beam with spot size varying in the range 1.1 $0.15 \mathrm{~cm}$ FWHM as a function of energy [23] and an intensity of about $10^{8}$ ions $/ \mathrm{s}$. The accelerator duty cycle was about $1 \mathrm{~s}$ spill and $2 \mathrm{~s}$ pause between spills (inter-spill). The INSIDE data acquisition system is capable of acquiring during the in-spill phase [24]; however in-spill coincidences were discarded in the analysis since, without additional processing, they add noise to the image due to the high random coincidence rate.

The target was a $15 \times 20 \times 15 \mathrm{~cm}^{3}$ PMMA phantom, with $1.18 \mathrm{~g} / \mathrm{cm}^{3}$ density, placed at the isocenter. To avoid background caused by residual activation, the phantom was replaced after each acquisition.

The two fields of a randomly chosen clinical carbon ion treatment plan of about $2.05 \mathrm{GyE}$ each (labelled $A$ and $B$ ) were delivered to irradiate the phantoms. Each irradiation was repeated twice ( $A 1$ and $A 2$, $B 1$ and $B 2$ ). The energy was in the $133.8-268.9 \mathrm{MeV} / \mathrm{u}$ interval. The duration of treatments $A 1,2$ and $B 1,2$ was $179 \mathrm{~s}$ and $165 \mathrm{~s}$, respectively, and the total number of delivered particles was $1.30 \cdot 10^{9}$ and $1.29 \cdot 10^{9}$ ions. The acquisition started at the beginning of the treatments and ended few minutes after the irradiations were stopped.

\subsection{FLUKA Monte Carlo Simulations for Carbon Ion Therapy}

The MC toolkit adopted by the INSIDE collaboration is FLUKA, which is widely used in the hadrontherapy community $[9,10]$. The $M C$ simulation ultimate goal is to produce a prior image to be compared with the experimental data. To this purpose, the following key aspects must be considered:

1. Treatment setup: the terminal part of the beamline and target geometry and materials must be accurately reproduced in the simulated setup. To match the measurements of the activity (and dose) in the transverse plane, it is also necessary to correctly reproduce in simulation the beam transverse shape. The beam dimension has been tuned to match the experimental measurement data of beam transversal width at the isocenter [23], which for carbon decreases with increasing beam energy in the 1.2-0.5 cm (FWHM) range.

2. Target model: in the case of phantoms with simple geometrical shapes, the target can be modelled describing its volumes and properties (chemical composition and density). If the target is a patient or an anthropomorphic phantom, the corresponding CT is imported in the geometry simulation. The FLAIR [25] graphical interface for FLUKA provides utilities to handle the CT import, its simplification in voxel-based volumes and regions and the material description. Tissues composition and densities are modelled on the basis of the CT Hounsfield Units as described in [26, 27].

3. Statistics: the target activation is orders of magnitude lower than the number of primary particles. If a small fraction of primaries is simulated, it can provide enough statistic to make the simulated dose converge to the planned one, but it produces an activity distribution that is too scarcely populated. In order to perform a significant comparison between measurements and simulations, it is instead necessary to simulate the full amount of planned particles. Presently this simulation requires about 
7.5 $10^{3} \mathrm{CPU}$ hours for each treatment field. Since tens of patients are treated daily in a typical center, this approach would be feasible only by means of an extended cloud-based parallelization. To increase the simulation speed and overcome this limitation, a biased simulation has been implemented, based on two steps. The first step simulates the hadron beam and the particle interactions within the target, including processes such as multiple scattering and nuclear interactions, like target and projectile fragmentation. The endpoint positions and times of the produced $\beta^{+}$ decaying isotopes are scored on a file. A fraction of 1/100 of the typical number of particles in a field of a fraction, about $2 \cdot 10^{9}$, are simulated in this step. In the second step the isotopes decay is simulated, using the output of the first step as generator and increasing the positron emission of the same factor previously used to reduce the statistics and speed up the simulation. Half-lives, beta energy spectrum endpoints for every relevant isotope [28] are taken into account, so as to generate a reliable momentum distribution of the emitted positrons. Positron annihilation and photon pair generation are then simulated.

4. Time pattern: the shape of in-beam PET images in accelerator facilities with pencil beam scanning depends on the acquisition time during and after the treatment as well as on the time pattern of the hadron delivery. Moreover, about ten isotopes are formed when PMMA is traversed by proton beams, with decay times varying from about $0.01 \mathrm{~s}(\mathrm{O} 13$ and $\mathrm{N} 12)$ to $1220 \mathrm{~s}(\mathrm{C} 11)$. It is therefore necessary to take into account these effects in the simulation. The simulation of the delivery of each spot (time, intensity and duration) is performed by integrating the information from the CNAO dose delivery system [29], where the beam is monitored by means of five ionization chambers. This allows to correctly reconstruct the images corresponding to different time intervals with respect to the beginning of the treatment.

5. Detector impact on image reconstruction: the MLEM tomographic reconstruction algorithm suffers from the missing projections because of the small acceptance angle, resulting in severe artefacts in $3 \mathrm{D}$ images. To correctly compare the prior image to the measurement, we implemented the detector model in the FLUKA simulation. The geometry and materials of the scintillator arrays are included in the second step of the simulation. Custom software has been developed to obtain the interaction time and deposited energy (hits) for each event in every detector element. Hits are then digitized and eventually merged so as to model the detector response. First, detector energy and time resolution are modeled with a gaussian smearing according to experimental data. Then, hits are sorted and the ones closer in time than three times the scintillator decay time are merged. Finally, the hits outside the energy window applied on the actual acquisition system are discarded. The digitized hits are then scanned for coincidences and images are reconstructed, exploiting the very same algorithms used to analyse experimental data. When comparing experimental and simulated activity images, the same selection on acquisition time is applied.

6. Simulation calibration: the simulation validation in our case is represented by the agreement between the experimental activity images and the simulated ones. As a general rule, the possible tuning of simulation parameters (i.e. its calibration) must be performed on an independent dataset. The INSIDE FLUKA simulation is calibrated 
comparing the beam shape [23] so as to match the dose distribution delivered to the patient in the simulation with the planned one, as described in [30].

7. Physics models: the models implemented in FLUKA feature a description of energy loss, scattering and interactions which lead to an excellent agreement of simulated dose profiles with those measured in several hadron therapy centers, both with proton and ion beams. In the very last stage of nuclear interactions $\beta^{+}$emitting isotopes, whose signal is detected with in-beam PET, are formed. Their production is then highly sensitive to the accuracy in the simulation of all the detailed reaction history. Still, the available experimental cross section data are well reproduced by FLUKA, with an uncertainty of about $10 \%$. The relevant aspects of FLUKA for particle therapy and treatment verification are thoroughly discussed in [31]. The agreement with experimental data is expected to improve thanks to the results of nuclear experiments dedicated to the measurement of nuclear fragmentation at energies relevant for hadrontherapy [32].

The simulation validation has been performed in previous works via the comparison with proton beam data acquired with a preliminary simple setup [13, 24], with homogeneous phantoms irradiated with monoenergetic pencil beams [33] and clinical-like treatments [34], with PMMA phantoms including air cavities [18] and finally with in-vivo data measured during two consecutive proton irradiations of a patient [30]. In-vivo results show that the average activity range agreement between simulation and measurements is within $1 \mathrm{~mm}$ and is comparable with measurement repeatability. The analysis on in vivo data is focused also on the acquisition time necessary to form images with this grade of reliability.

The implemented simulation procedure allows the production of a reliable prior image, allowing an absolute comparison of measured and simulated 3D image series of the activity shape, during and after the irradiation, for any selected temporal interval. Thanks to the details implemented in MC simulation and data post-processing, this tool is also useful to reliably estimate the expected improvements in case of detector and/or electronics upgrade, active area enlargement or reconstruction algorithm optimizations.

\subsection{Image comparison}

PET images were reconstructed every $10 \mathrm{~s}$, starting from the beginning of the treatment $(\mathrm{t}=0)$, so as to analyse the time evolution of the activity distributions, with the goal to assess the minimum acquisition time required to provide reliable images. Acquired and simulated images were processed with a median filter of $5 \mathrm{~mm}$ radius, to smooth out the noise caused by low statistic. Furthermore, images were equalized before the comparison [35] and normalized to their maximum intensity.

The beam irradiates only a region of the transversal section of the FOV; hence only the voxels with $x, y$ coordinates (fig. $1 \mathrm{~b}$ ) corresponding to the transversal surface through which the beam was delivered were taken into account in image comparison, as in previous studies by other collaborations [36, 37].

Three methods were investigated to measure the agreement between simulation and data. 


\subsubsection{Pearson Correlation Coefficient}

The Pearson Correlation Coefficient (PCC) provides an overall agreement index (0-1) between the image intensities. According to [38], it was evaluated as follows:

- Image intensity profiles were calculated for each $1 \times 1(x-y)$ region along the $z$ axis.

- For each profile, a ROI corresponding to the region between $95 \%$ and $25 \%$ of the activity profile maximum, moving towards the distal fall-off, was selected.

- Once the full 3D ROI is defined, the PCC is then calculated as follows:

$$
P C C=\frac{\sum_{i=1}^{n}\left(x_{i}-\bar{x}\right)\left(y_{i}-\bar{y}\right)}{\sqrt{\sum_{i=1}^{n}\left(x_{i}-\bar{x}\right)^{2}} \sqrt{\sum_{i=1}^{n}\left(y_{i}-\bar{y}\right)^{2}}}
$$

where $\mathrm{x}_{\mathrm{i}}\left(\mathrm{y}_{\mathrm{i}}\right)$ and $\bar{x}(\bar{y})$ are the intensity of the ith voxel and the average intensity in the ROI of the first (second) image, respectively.

\subsubsection{Beam-Eye View}

The Beam-Eye View (BEV) method consists in the comparison of the activity distal edges of each image along the $z$ axis, i.e. beam-view direction, as suggested in [36-39]. To perform this comparison, a 3D iso-activity connected surface that defines the irradiated volume with an activity above a threshold was calculated for each image, as follows:

- First, a low threshold was defined with respect to the image maximum, so as to exclude the image noise, and only the image voxels with intensity over the threshold were considered. The largest connected region survived to the cut was then selected.

- An erosion (3 $\mathrm{mm})$ and a dilation $(5 \mathrm{~mm})$ filter were then applied to the surface, to mildly smooth out the surface and include any small but relevant region close to the main one.

- The low threshold was then re-applied as in the first step, so as to select an iso-activity surface.

- For each $x, y$ position on the beam delivery surface, the distance between the distal edge of the two images to be compared was evaluated.

- The distribution of the so-calculated beam-view distance between the distal edges was then analysed, so as to evaluate the activity range agreement between the two images. 


\subsubsection{Overall View}

The phantom had to be replaced after each irradiation to discard residual activity, a procedure that brings an unwanted uncertainty on phantom positioning. It is then necessary to develop a method less sensitive to the absolute phantom position. The Overall View approach (OV) is also based on the calculation of iso-activity surfaces, but instead of relying only on the distal edge and considering the beam direction as preferential, it provides an overall distance evaluation between the two contours. Because of this non-preferential direction, the OV distance distribution is far less sensitive to the uncertainty in the phantom positioning with respect to detector than the BEV analysis. The OV comparison was performed as follows:

- Iso-activity surfaces were calculated with the same procedure as in the BEV analysis.

- For each pixel in one image surface, the nearest pixel was found on the other surface and their cartesian distance was calculated.

- The distribution of the distances was studied to evaluate the agreement between the irradiated volumes of the images to be compared.

In this work, the average of the range difference (BEV) or surface distance (OV) distributions, together with their FWHM, were selected as system performance indicators (an example is shown in fig. 2).

\section{Results}

The data acquisition system performed as expected for each run, both during inter-spill and in-spill, as shown in fig. 3 . The time delay associated to the image reconstruction was about $5 \mathrm{~s}$.

The image series of the activity evolution were reconstructed considering the coincidences acquired since the treatment start, increasing the integration time in $10 \mathrm{~s}$ long steps. Some examples of the reconstructed images at different times are shown in fig. 4.

The PCC reaches 0.90 after $180 \mathrm{~s}$ for both $\mathrm{A} 1$ vs $\mathrm{A} 2$ and $\mathrm{B} 1 \mathrm{vs} \mathrm{B} 2$ data comparison, i.e at the end of the delivery for $A$ fields and 15 seconds after the end of treatment for $B$ fields (fig $5)$. When comparing the data images with the corresponding images derived from Monte Carlo simulations, the same indicators range between 0.85 and 0.87 at $180 \mathrm{~s}$, while 0.90 is reached about $40 \mathrm{~s}$ later.

It is also necessary to check that the agreement between data and simulation is not dependent on the bias applied in the simulation. Consequently a simulation of the B1 field was performed with a bias ten times lower (i.e. 1/10 of the primary particles were simulated in the first step instead of 1/100). This dataset, called $B 1_{1 / 10}$, was compared to the experimental B1 acquisition with the PCC method. As shown in fig. 5c, there is no significant change in the agreement with the experimental data.

The Monte Carlo simulation can also be exploited to extract information not directly observable otherwise. This is an insightful operation for instance when planning a measurement or in order to better understand its results. As an example, we show in fig 6 
the $z$ coordinate of the positron annihilations inside the phantom for treatments $A$ and $B$; the contributions of the most relevant isotopes are shown. The analysis of annihilations occurring during the treatment shows, interestingly, that the contribution of fast isotopes such as B8 is quite significant.

The BEV and OV methods rely on the extraction of an iso-activity contour surface for each image. The threshold applied to extract the contour was chosen to be slightly above the image background, so as to extract a contour of the activated area as wide as possible. The optimal thresholds is $20 \%(12 \%)$ of the image maximum for data vs data and data vs simulation comparisons, respectively. The difference is related to the different amount of noise in the two images: the activity shape in the data image is enhanced when equalised to the less noisy simulated image.

The BEV method (fig. $2 \mathrm{a}$ and 7 ) results indicate that the agreement in range along the beam direction at the end of the treatment (180 s) when comparing $A 1,2$ fields $(B 1,2)$ is within 2.9 $\mathrm{mm}(0.6 \mathrm{~mm})$, while the FWHM of the difference distributions is about $12.5 \mathrm{~mm}$. For longer acquisition time, the agreement on the average range is in general within $1 \mathrm{~mm}$. After the treatment end, the FWHM decreases in the 8-12 $\mathrm{mm}$ range. Very similar results are obtained when comparing each acquisition with its corresponding Monte Carlo simulation: at treatment end the average range difference is $1 \mathrm{~mm}$, while the FWHM of the distribution is in the $10-13 \mathrm{~mm}$ interval. Although the $A$ and $B$ fields have a similar total particle number (1.3.10 $10^{9}$ ions), differences in range agreement as a function of time for $A 1$ vs $A 2$ and $B 1$ vs B2 may be caused by the different temporal and spatial distribution of the particles in the $A$ and B fields. Systematic effects in this case are also caused by the uncertainty in the phantom positioning, obtained with manual alignment with the isocenter laser markers in the treatment room, with a precision of about $2 \mathrm{~mm}$.

For the OV method the agreement (fig. $2 \mathrm{~b}$ and 8 ) is numerically lower, with a sub-millimetric average after $100 \mathrm{~s}$ for every pair of images compared (i.e., less than one pixel) and a FWHM of less than $5 \mathrm{~mm}$.

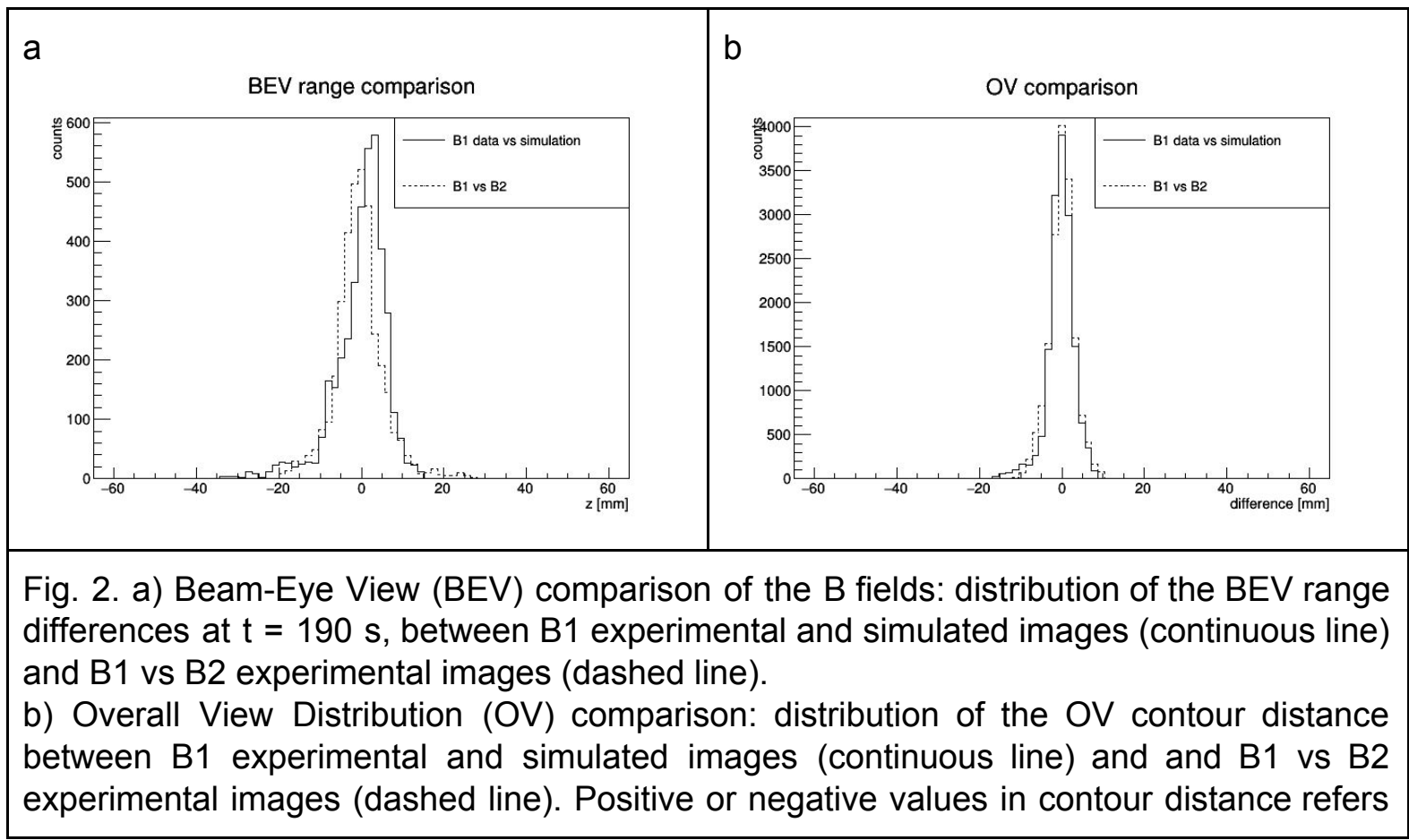


to the pixel of the first contour being internal or external with respect to the second contour.

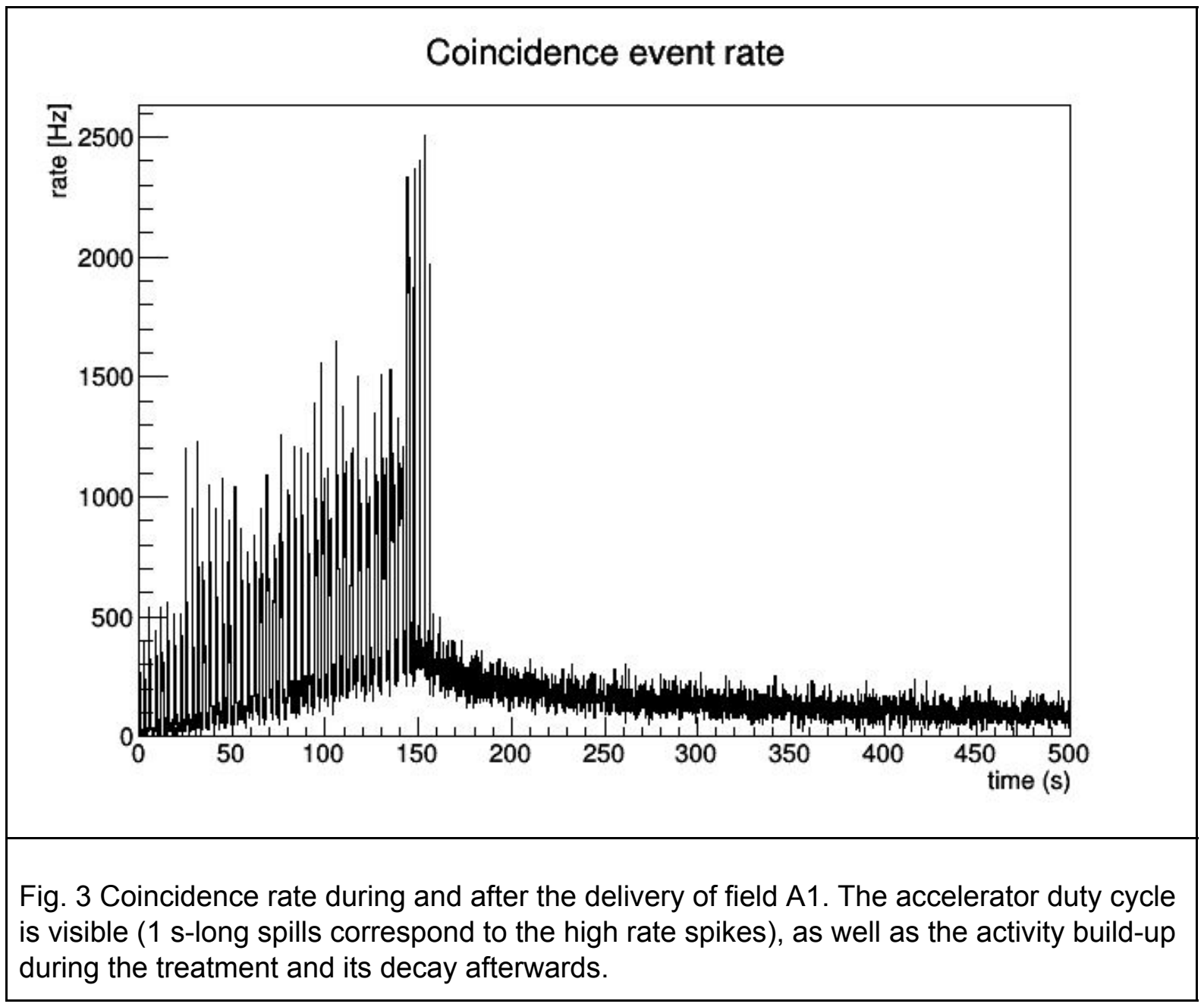




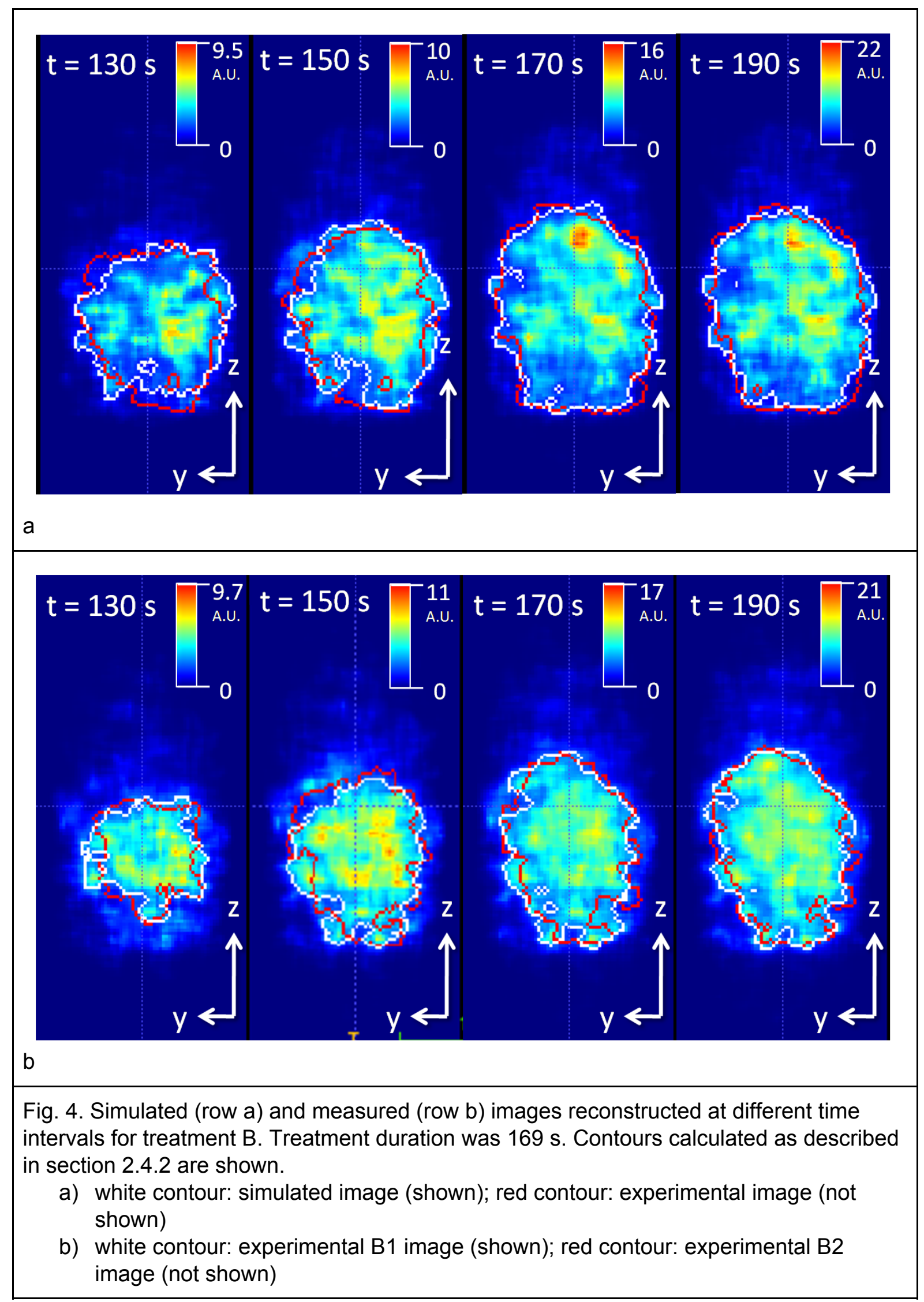



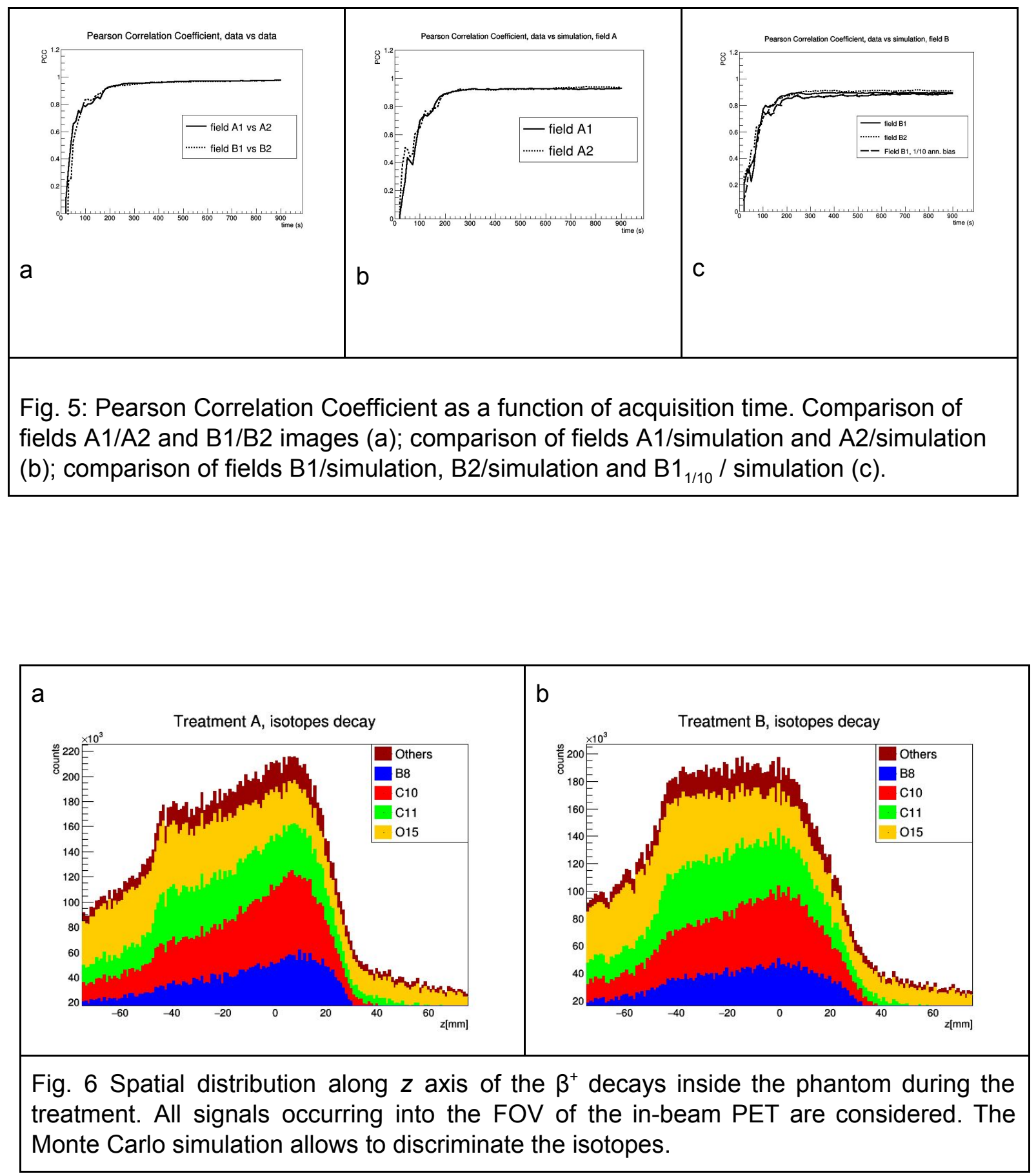

\begin{tabular}{|l|l|}
\hline$a$ & $b$
\end{tabular} 


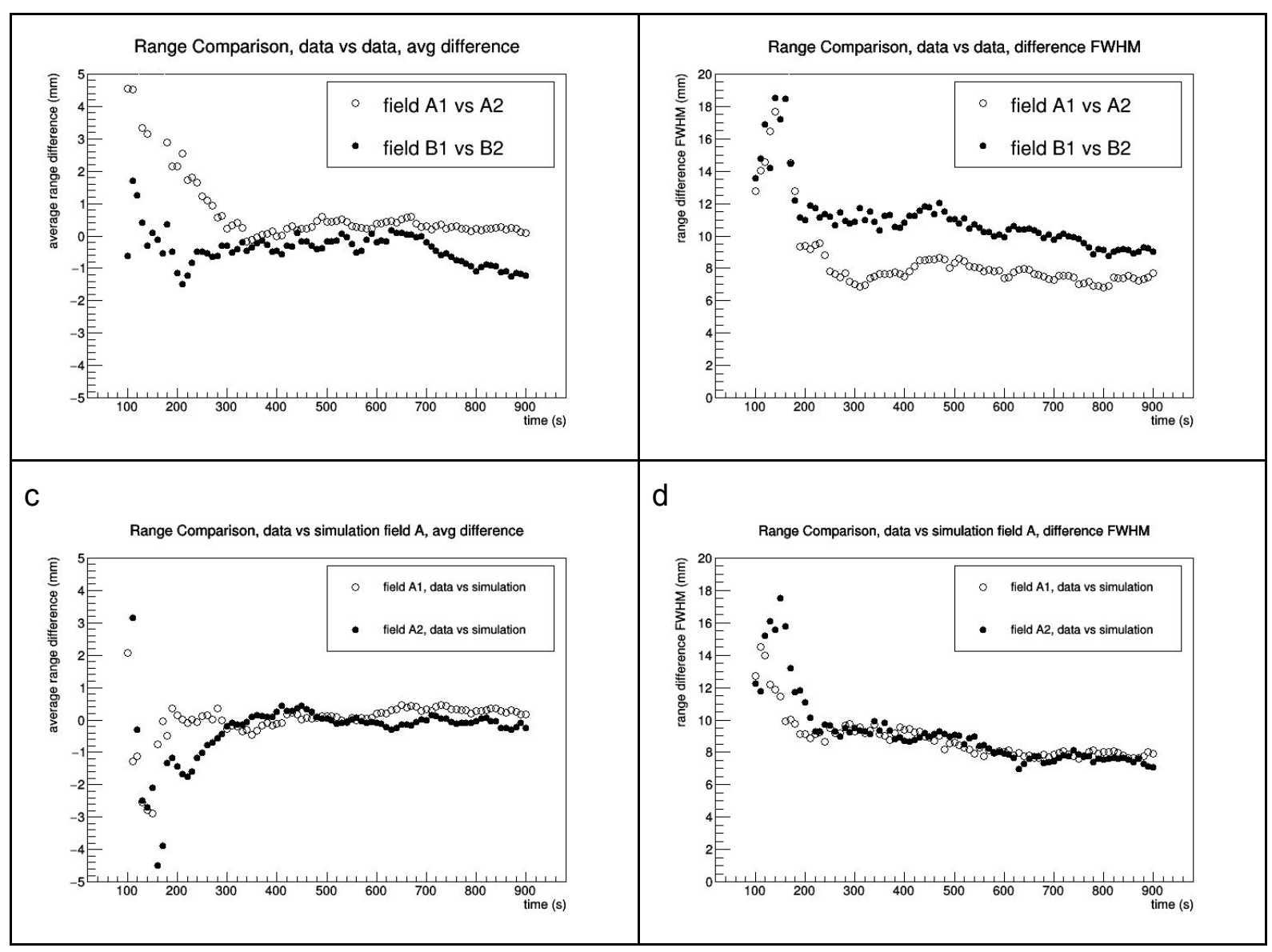



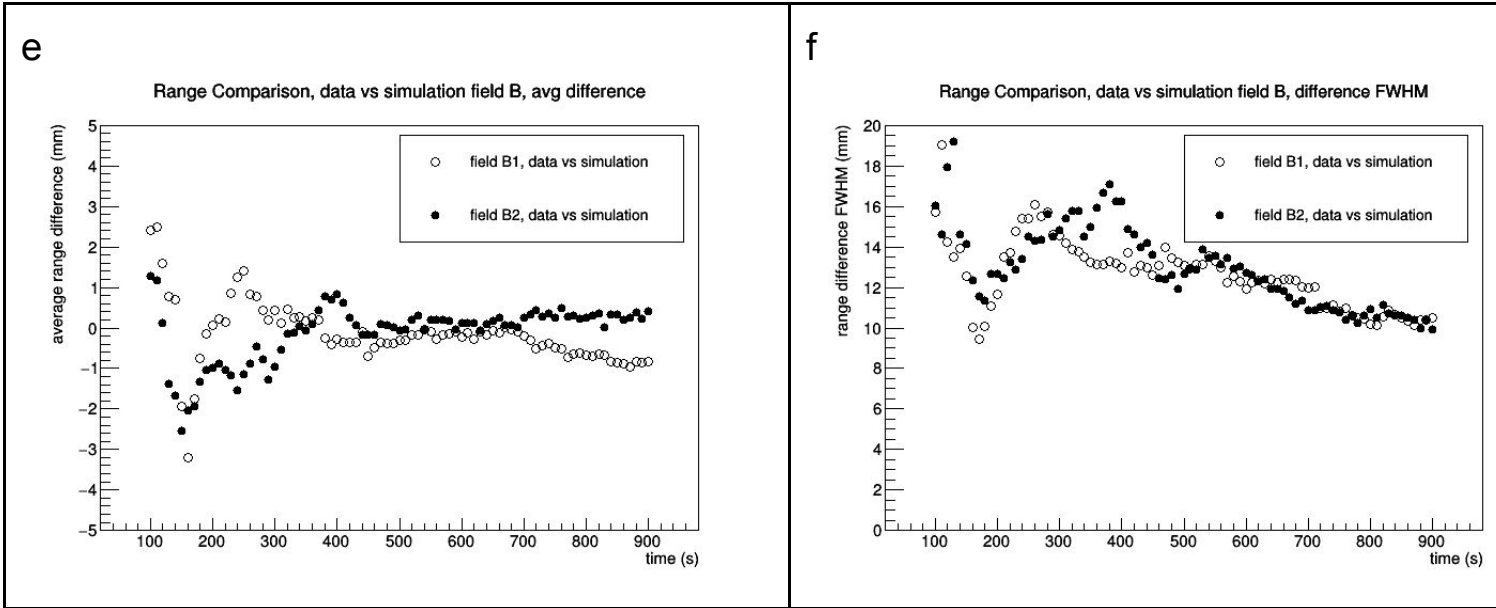

Fig. 7: Beam's Eye View, average range difference and FWHM of the difference distribution as a function of time. Fields $A 1$ vs $A 2$ and $B 1$ vs $B 2(a, b)$; fields $A 1$ vs simulation and $A 2$ vs simulation (c,d); fields $B 1$ vs simulation and $B 2$ vs simulation $(e, f)$.

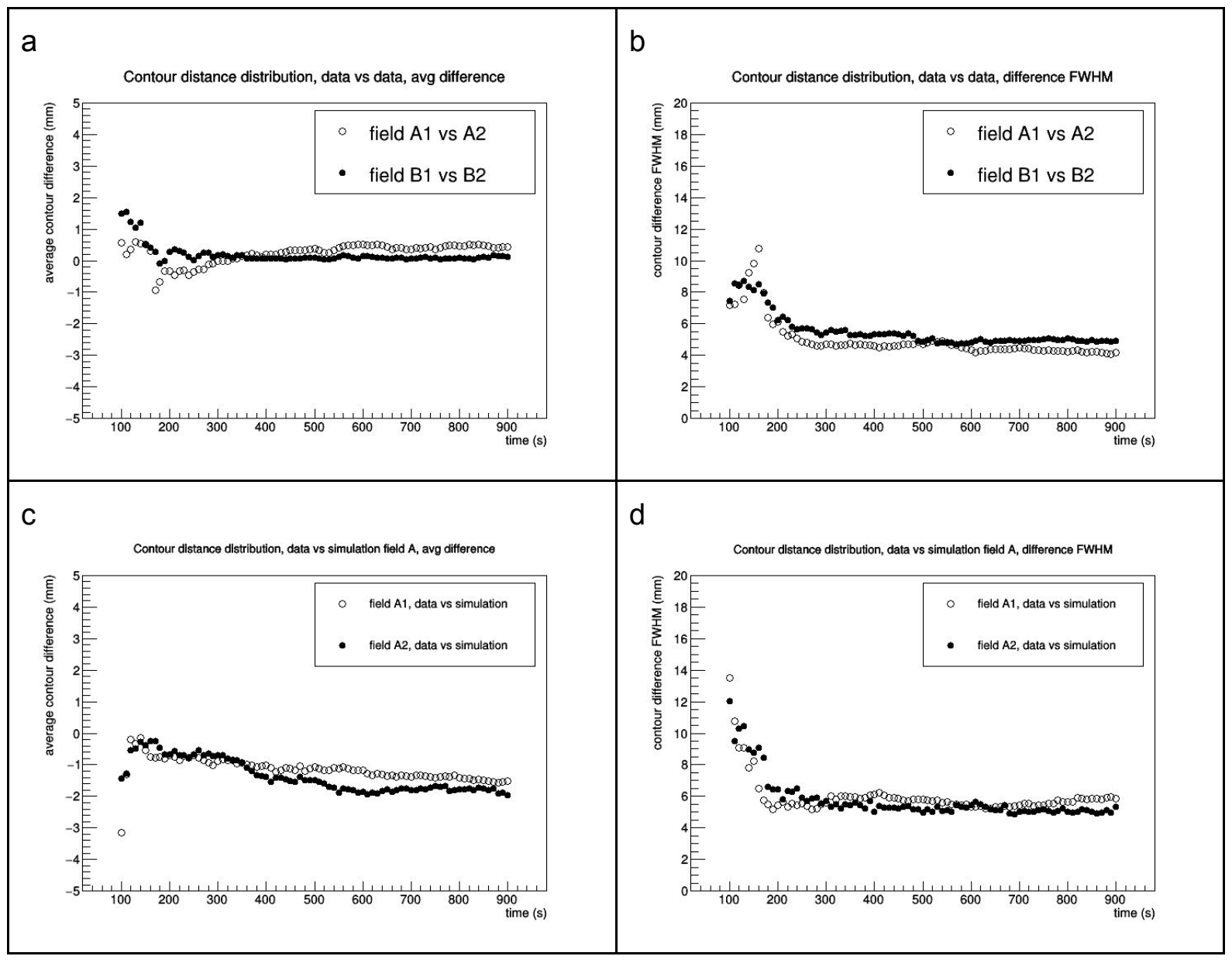



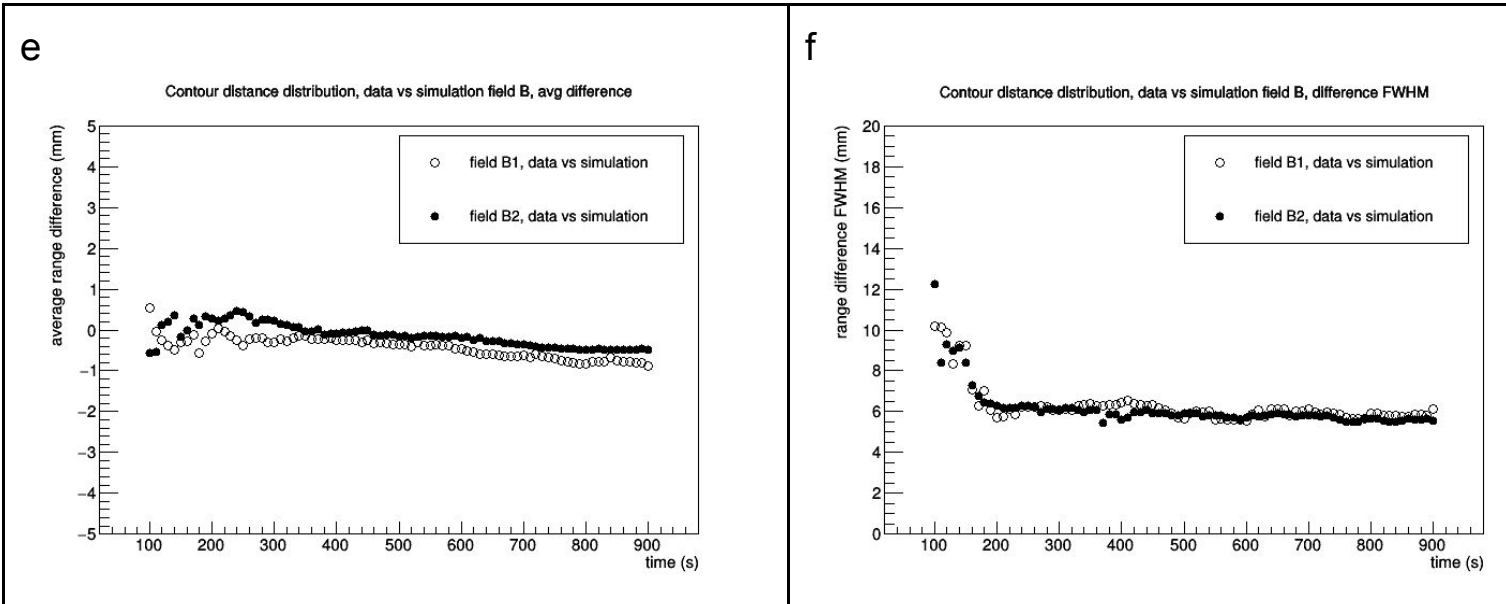

Fig. 8: Overall View, average contour distance and FWHM of the distribution as a function of time. Fields $A 1$ vs $A 2$ and $B 1$ vs $B 2(a, b)$; fields $A 1$ vs simulation and $A 2$ vs simulation $(c, d)$; fields $B 1$ vs simulation and B2 vs simulation $(e, f)$.

\section{Discussion and conclusion}

The SiPM-based in-beam PET of the INSIDE project has been tested at CNAO during the irradiation of PMMA phantoms with carbon ion beams. Two actual treatment plan fields of about 2 GyE each were delivered, with each fraction repeated twice.

The data acquisition system performed as expected, both during the beam delivery (in-spill) and during the pauses between the spills (inter-spill). In-spill coincidences were discarded so as to avoid the contribution of random coincidences (about $40 \%$ at the treatment end). Refinements of the analysis system to filter the in-spill data random coincidences are beyond the scope of this work; nevertheless some promising off-line results were already obtained with a prototype [40] for irradiation with proton beams.

The repetition of the irradiations allowed a study of the acquisition time needed to collect enough coincidences so that the images converge to a reproducible result. Since the time-tagged coincidence acquisition of the INSIDE system allows to reconstruct images for different time intervals, we could show that, within at most $20 \mathrm{~s}$ after the end of $170 \mathrm{~s}$ long carbon ions therapy fields delivery, the average range agreement of repeated irradiations is about 1-2 mm.

A FLUKA-based Monte Carlo simulation, that implements the figures of merit of the CNAO dose delivery system (pencil beam scanning, particle energy distribution, beam width as a function of the energy, time structure), has been developed. The simulation uses as input the data log of the accelerator, to enforce the correct space and time distribution of the pencil beam. The simulated layout includes the detector geometry and its actual time and energy resolution; also, the images from simulated data are reconstructed with the same algorithm used for the experimental ones. The simulation is based on two steps, so as to optimise the runtime without compromising the accuracy.

The acquisitions analysed were reproduced with the Monte Carlo simulation. Their agreement with the experimental data sets, using the PCC, BEV and OV methods described 
in section 2.4, is comparable to the agreement between repeated data sets, all along the acquisition time.

The results on homogeneous phantoms, in terms of acquisition and measurements repeatability, are comparable with the ones already obtained with proton beams $[5,18,30]$. This is promising towards further tests of the scanner, that will soon culminate with its in-vivo extensive test and characterization with both proton and carbon ion beams.

It was also shown that with the INSIDE in-beam PET, for the two therapeutic fields considered in this work, a short after-treatment acquisition (about $20 \mathrm{~s}$ ) is necessary for collecting enough data to obtain accurate results. This after-treatment time is shorter than normal intervals occurring between the delivery of two consecutive fields in the clinical practice and therefore compatible with clinical settings. More case studies will be necessary to consolidate these results and therefore to define an actual clinical routine. On the other hand, in order to achieve an effective treatment monitoring with a response faster than the irradiation time, it should be necessary to extend the detector geometrical acceptance, so as to increase the coincidence detection rate and hence provide more statistics to the reconstruction algorithm in a shorter time, improving the response stability. An alternative and more powerful approach could based on imaging algorithm optimisation and an accurate image post-processing, so as to extract the range information from the decay of short-lived isotopes (such as Boron-8 and Nitrogen-12) and increase the range monitoring accuracy [41]. The latter approach, in principle achievable with the INSIDE PET scanner because of its in-spill acquisition capability, is a key to enable fast-isotopes measurements in synchrotrons and is under study.

The developed Monte Carlo simulation shows results comparable within the repeatability of experimental acquisitions: the calculation of a prior image with the implemented simulation tool is therefore ready for use in in-vivo tests.

In conclusion, the results presented in this work focusing on carbon ions online treatment monitoring are a further step for the INSIDE scanner towards a clinical trial, which will start at CNAO in 2018, with the aim of longitudinal monitoring of about 20 patients, treated with proton or carbon ion beams.

\section{References}

1. Knopf, AC., et al. "In vivo proton range verification: a review." Phys Med Biol 58.15 (2013): R131.

2. Camarlinghi, N., et al. "An in-beam PET system for monitoring ion-beam therapy: test on phantoms using clinical $62 \mathrm{MeV}$ protons." J Instrum 9.04 (2014): C04005.

3. Kraan, A., et al., "Online monitoring for proton therapy: A real-time procedure using a planar PET system." Nucl Instrum Meth A 786 (2015): 120-126.

4. Rossi, S. "The status of CNAO." Eur Phys J Plus 126.8 (2011): 1-39.

Mirandola, Alfredo, et al. "Dosimetric commissioning and quality assurance of scanned ion beams at the Italian National Center for Oncological Hadrontherapy." Medical physics 42.9 (2015): 5287-5300.

5. Ferrero, V., Fiorina E., Morrocchi M., Pennazio F., et al. "Online proton therapy monitoring: clinical test of a Silicon-photodetector-based in-beam PET", Scientific Reports, 8.1 (2018):4100

6. Zhu, Xuping, and Georges El Fakhri. "Proton therapy verification with PET imaging." Theranostics 3.10 (2013): 731.

7. Parodi, Katia. "PET monitoring of hadrontherapy." Nuclear Medicine Review 15.C (2012): 37-42.

8. Attanasi, F., et al. "Extension and validation of an analytical model for in vivo PET verification of proton therapy - a phantom and clinical study." Physics in medicine and biology 56.16 (2011): 5079.

9. T.T. Bohlen, F. Cerutti, M.P.W. Chin, A. Fasso', A. Ferrari, P.G. Ortega, A. Mairani, P.R. Sala, G. Smirnov, and V. Vlachoudis, "The FLUKA Code: Developments and Challenges for High Energy and 
Medical Applications", Nuclear Data Sheets 120, 211-214 (2014)

10. A. Ferrari, P.R. Sala, A. Fasso", and J. Ranft, "FLUKA: a multi-particle transport code", CERN-2005-10 (2005), INFN/TC_05/11, SLAC-R-773

11. K. Parodi, "On-and off-line monitoring of ion beam treatment", Nuclear Instruments and Methods in Physics Research Section A: Accelerators, Spectrometers, Detectors and Associated Equipment 809 (2016) 113-119.

12. Shakirin, G., Braess, H., Fiedler, F., Kunath, D., Laube, K., Parodi, K., ... \& Enghardt, W. (2011). "Implementation and workflow for PET monitoring of therapeutic ion irradiation: a comparison of in-beam, in-room, and off-line techniques." Physics in medicine and biology, 56(5), 1281.

13. Pennazio, F., Attili, A., Battistoni, G., Belcari, N., Bisogni, M. G., Camarlinghi, N., ... \& Cirrone, G. A. P. (2015). "A Study of Monitoring Performances with the INSIDE System." Acta Physica Polonica A, 127(5), 1468-1470.

14. Enghardt, W., Crespo, P., Fiedler, F., Hinz, R., Parodi, K., Pawelke, J., \& Pönisch, F. (2004). "Charged hadron tumour therapy monitoring by means of PET." Nuclear Instruments and Methods in Physics Research Section A: Accelerators, Spectrometers, Detectors and Associated Equipment, 525(1), 284-288.

15. Sportelli, G., Belcari, N., Camarlinghi, N., Cirrone, G. A. P., Cuttone, G., Ferretti, S., ... \& Straub, K. (2013). "First full-beam PET acquisitions in proton therapy with a modular dual-head dedicated system." Physics in medicine and biology, 59(1), 43.

16. Zhu, X., España, S., Daartz, J., Liebsch, N., Ouyang, J., Paganetti, H., ... \& El Fakhri, G. (2011). Monitoring proton radiation therapy with in-room PET imaging. Physics in medicine and biology, 56(13), 4041.

17. Bauer, J., Unholtz, D., Sommerer, F., Kurz, C., Haberer, T., Herfarth, K., ... \& Parodi, K. (2013). Implementation and initial clinical experience of offline PET/CT-based verification of scanned carbon ion treatment. Radiotherapy and Oncology, 107(2), 218-226.

18. M. G. Bisogni, A. Attili, G. Battistoni, N. Belcari, P. Cerello, S. Coli, A. Del Guerra, A. Ferrari, V. Ferrero, E. Fiorina, et al., "Inside in-beam positron emission tomography system for particle range monitoring in hadrontherapy", Journal of medical imaging 4 (1) (2017) 011005-011005.

19. Rolo, M. D., et al. "Tofpet asic for pet applications." Journal of Instrumentation 8.02 (2013): C02050.

20. Vardi, Yehuda, L. A. Shepp, and Linda Kaufman. "A statistical model for positron emission tomography." Journal of the American statistical Association 80.389 (1985): 8-20.

21. Moehrs, Sascha, et al. "Multi-ray-based system matrix generation for 3D PET reconstruction." Physics in Medicine \& Biology 53.23 (2008): 6925.

22. Camarlinghi, N., et al. "An in-beam PET system for monitoring ion-beam therapy: test on phantoms using clinical $62 \mathrm{MeV}$ protons." Journal of Instrumentation 9.04 (2014): C04005.

23. A. Mirandola, S. Molinelli, G. Vilches Freixas, A. Mairani, E. Gallio, D. Panizza, S. Russo, M. Ciocca, M. Donetti, G. Magro, S. Giordanengo, R. Orecchia, "Dosimetric commissioning and quality assurance of scanned ion beams at the italian national center for oncological hadrontherapy", Medical Physics 42 (9) (2015) 5287-300.

24. Piliero, M. A., et al. "Performance of a fast acquisition system for in-beam PET monitoring tested with clinical proton beams." Nuclear Instruments and Methods in Physics Research Section A: Accelerators, Spectrometers, Detectors and Associated Equipment 804 (2015): 163-166.

25. Vlachoudis, Vasilis. "FLAIR: a powerful but user friendly graphical interface for FLUKA." Proc. Int. Conf. on Mathematics, Computational Methods \& Reactor Physics (M\&C 2009), Saratoga Springs, New York. 2009.

26. Parodi, Katia, et al. "PET/CT imaging for treatment verification after proton therapy: a study with plastic phantoms and metallic implants." Medical physics 34.2 (2007): 419-435.

27. Schneider, Wilfried, Thomas Bortfeld, and Wolfgang Schlegel. "Correlation between CT numbers and tissue parameters needed for Monte Carlo simulations of clinical dose distributions." Physics in Medicine \& Biology 45.2 (2000): 459. 
28. Fiedler, F., Kunath, D., Priegnitz, M., \& Enghardt, W. (2012). Online irradiation control by means of PET. In Ion Beam Therapy (pp. 527-543). Springer Berlin Heidelberg.

29. S. Giordanengo et al. "The CNAO dose delivery system for modulated scanning ion beam radiotherapy" Med. Phys. 42 (263), Jan 2015 - doi: 10.1118/1.4903276

30. Fiorina, E., et al. "Monte Carlo simulation tool for online treatment monitoring in hadrontherapy with in-beam PET: A patient study." Physica Medica (2018) doi.org/10.1016/j.ejmp.2018.05.002-.

31. Battistoni, Giuseppe, et al. "The FLUKA code: an accurate simulation tool for particle therapy." Frontiers in oncology 6 (2016): 116.

32. Battistoni, Giuseppe, et al. "The FOOT (Fragmentation Of Target) Experiment." PoS (2017): 023.

33. Fiorina, E., and INSIDE collaboration. "An integrated system for the online monitoring of particle therapy treatment accuracy." Nuclear Instruments and Methods in Physics Research Section A:

Accelerators, Spectrometers, Detectors and Associated Equipment 824 (2016): 198-201.

34. Ferrero, V., and INSIDE collaboration. "The INSIDE project: on-line monitoring and simulation validation with the in-beam PET scanner." Journal of Physics: Conference Series. Vol. 841. No. 1. IOP Publishing, 2017.

35. Paranjape, Raman B. "Fundamental enhancement techniques." Handbook of medical imaging. Academic Press, Inc., 2000.

36. Min, C. H., Zhu, X., Grogg, K., El Fakhri, G., Winey, B., \& Paganetti, H. (2015). "A recommendation on how to analyze in-room $\mathrm{PET}$ for in vivo proton range verification using a distal PET surface method." Technology in cancer research \& treatment, 14(3), 320-325.

37. Frey, K., Unholtz, D., Bauer, J., Debus, J., Min, C. H., Bortfeld, T., ... \& Parodi, K. (2014). "Automation and uncertainty analysis of a method for in-vivo range verification in particle therapy." Physics in medicine and biology, 59(19), 5903.

38. P. Kuess, W. Birkfellner, W. Enghardt, S. Helmbrecht, F. Fiedler, D. Georg, "Using statistical measures for automated comparison of in-beam PET data" Medical Physics 39 (10) (2012) 5874-5881.

39. Nishio, T., Miyatake, A., Ogino, T., Nakagawa, K., Saijo, N., \& Esumi, H. (2010). "The development and clinical use of a beam ON-LINE PET system mounted on a rotating gantry port in proton therapy." International Journal of Radiation Oncology * Biology* Physics, 76(1), 277-286.

40. M. Piliero, F. Pennazio, M. Bisogni, N. Camarlinghi, P. Cerello, A. Del Guerra, V. Ferrero, E. Fiorina, G. Giraudo, M. Morrocchi, et al., "Full-beam performances of a pet detector with synchrotron therapeutic

proton beams", Physics in medicine and biology 61 (23) (2016) N650.

41. P. Dendooven, H. Buitenhuis, F. Diblen, P. Heeres, A. Biegun, F. Fiedler, M. Van Goethem, E. Van der Graaf, S. Brandenburg, "Short-lived positron emitters in beam-on PET imaging during proton therapy", Physics in medicine and biology 60 (23) (2015) 8923. 